\title{
THE COMPLEX OF CURVES ON NON-ORIENTABLE SURFACES
}

\author{
MARTIN SCHARLEMANN
}

Let $\mathscr{P} \mathscr{F}(M) \subset \mathscr{P}\left(R_{+}^{\mathscr{S}}-0\right)$ denote the projectivized space of measured foliations on a compact surface $M$ with negative Euler characteristic, as studied by Thurston [3], and let $\mathscr{P} \mathscr{F}_{0}(M)$ denote the subspace consisting of those foliations in which each boundary component is a leaf (containing at least one singularity). If $M$ is the sum of $g$ tori, $d$ disks and $p$ projective planes, then $\mathscr{P} \mathscr{F}_{0}(M) \cong S^{6 g+3 p+2 d-7}$ and $\mathscr{P} \mathscr{F}(M)$ is the join of $\mathscr{P} \mathscr{F}_{0}(M)$ to a $(d-1)$-simplex.

There is a subcomplex $X_{0}$ in $\mathscr{P} \mathscr{F}_{0}(M)$ whose $(n-1)$-simplices consist of foliations obtained by "enlarging" $n$ disjoint simple, closed, connected curves $C_{1}, \ldots, C_{n}$, none of which bounds a disk, or is boundary parallel, and no two of which bound an annulus. A subcomplex $X$ of $\mathscr{P} \mathscr{F}(M)$ can be defined in much the same way, except that we allow proper arcs as well as simple closed curves.

The complexes $X_{0}$ and $X$ have an interesting structure in their own right; since they are preserved under diffeomorphism, their structure gives geometric insight into the structure of automorphisms of $M$. Unfortunately, their structure is quite complex, since $X_{0}$ and $X$ are rarely locally finite.

Floyd and Hatcher [2] have constructed all the low-dimensional examples (and one of dimension 5) in the cases where $M$ is orientable. The purpose of the present paper is two-fold. First, we show that when $M$ is not orientable, $X_{0}$ has some surprising qualities not found when $M$ is orientable. Secondly, we construct the complexes for those remaining cases in which the foliation space is of dimension one or two. The case of the 3-punctured $R P^{2}$ is particularly interesting and is the subject of $\S 3$. In this case $X_{0}$ is the complex obtained from the tetrahedron by repeated star subdivision of all the faces but no edges. In the resulting complex the vertices represent all the 1-sided curves, and an interior point of each edge all the 2-sided curves.

I would like to thank David Chillingworth for his very helpful comments on the original manuscript.

\section{One-sided versus two-sided curves}

The core of a Mbbius band in $M$ and the (two-sided) edge of the band enlarge to the same foliation and hence represent the same point in $\mathscr{P} \mathscr{F}(M)$. We shall say that this point corresponds to a one-sided curve, while a point corresponding to a twosided curve will mean also that the point does not come from a one-sided curve.

If $M$ is orientable, the vertices of $X_{0}$ (representing connected curves) are dense in $\mathscr{P} \mathscr{F}_{0}(M)[1 ; 6 . I V .2]$. The proof uses the orientability of $M$ quite heavily. In fact, we have the following.

Received 4 November, 1980; revised 16 February, 1981.

During the preparation of this work, the author was supported in part by an NSF grant.

[J. LONDON MATH. SOC. (2), 25 (1982), 171-184] 
THEOREM 1.1. If $M$ is not orientable the vertices of $X_{0}$ corresponding to one-sided curves are isolated.

Proof. Let $C$ be a one-sided curve in $M$. Since $\mathscr{X}(M)<0, M$ contains a twicepunctured $R P^{2}$, denoted $N$, containing $C$, such that neither boundary component of $N$ is null-isotopic in $M$.

Figure 1 shows $C$ lying in $N$, viewed as a 2 -holed disk with antipodal boundary points identified. The boundary components of $N$ are labelled $s_{1}$ and $s_{2}$.

A third curve $s_{3}$, equivalent in $\mathscr{P} \mathscr{F}(M)$ to $R P^{1} \subset R P^{2}$, is also shown.

The topology on $\mathscr{P} \mathscr{F}(M)$ is that inherited from $\mathscr{P}\left(R_{+}^{\mathscr{S}}-0\right)$, where $\mathscr{S}$ is the collection of closed curves, by assigning to a foliation $\mathscr{F}$ the coordinate $\int_{s} \mathscr{F}$ on the axis corresponding to $s$. If $\mathscr{F}$ is obtained by enlarging a curve $\bar{s}$, then instead use the minimal number of intersection points $i(\bar{s}, s)$ of $\bar{s}$ with $s$. In particular, the coordinates of $C$ in $R_{+}^{s_{1}} \times R_{+}^{s_{2}} \times R_{+}^{s_{3}}$ are $(0,0,2)$. In fact, any connected simple closed curve in $M$ whose coordinates $\left(x_{1}, x_{2}, x_{3}\right)$ have $x_{1}, x_{2}<\frac{1}{2} x_{3}$ is isotopic to $C$ (or to a parallel 2-sided curve). Indeed, suppose the coordinates of a curve $s_{4}$ have $x_{1}, x_{2}<\frac{1}{2} x_{3}$. Then, after isotoping $s_{4}$ to minimize its intersection with $s_{1}, s_{2}$, and $s_{3}$, the intersection of $s_{4}$ with $N$ is as in Figure 2, where the end of each arc is identified with the end $\left(a_{1}+a_{2}+2 a_{3}\right) / 2$ places to the right (or left). Note that $x_{1}=a_{1}, x_{2}=a_{2}$, and $x_{3}=a_{1}+a_{2}+2 a_{3}$ is even. Since $x_{3}>2 x_{1}, 2 a_{3}>\left(a_{1}-a_{2}\right)$. Then

Let

$$
2 a_{3}+\left(a_{2}-a_{1}\right)=2 y>0 .
$$

$$
z= \begin{cases}y / 2 & (y \text { even }) \\ (y-1) / 2 & (y \text { odd })\end{cases}
$$

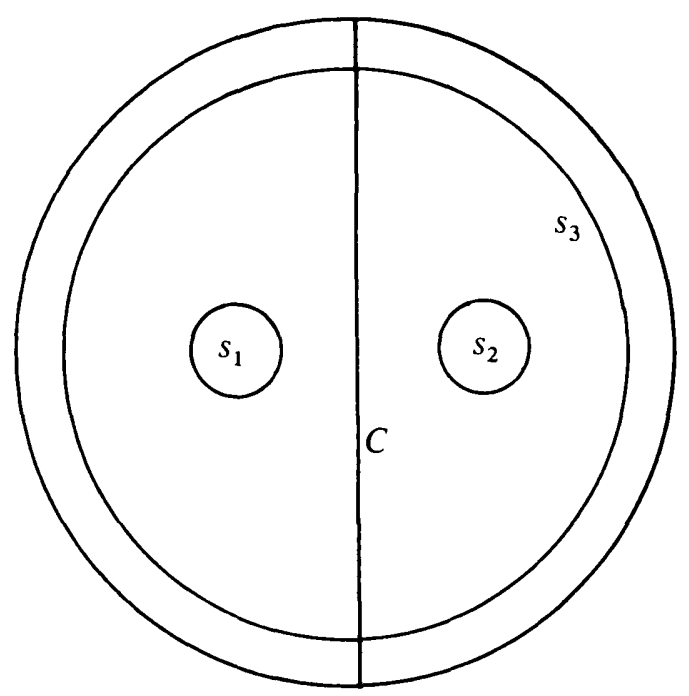

Figure 1. 


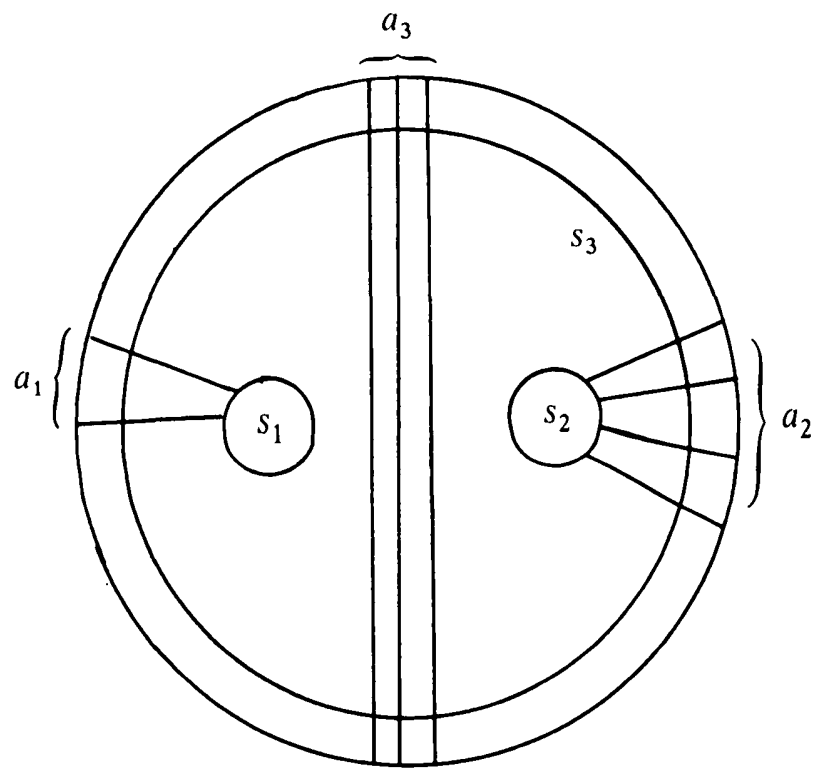

Figure 2.

We have $a_{1}+2 z=a_{2}+2\left(a_{3}-z\right)$ in the first case or $a_{1}+2 z=a_{2}+2\left(a_{3}-z-1\right)$ in the second. Thus there is a simple closed curve in $s_{4}$ for which $a_{1}=a_{2}=0$ and $a_{3}=1$ or 2 (depending on the parity of $2 z$ ). But $s_{4}$ is connected, and so $x_{1}=x_{2}=0$ and $x_{3}=2$ or 4 .

As a counterpoint to Theorem 1.1 we have the following.

Proposition 1.2. The closure of the vertices in $X_{0}$ corresponding to one-sided curves contains all those corresponding to two-sided curves.

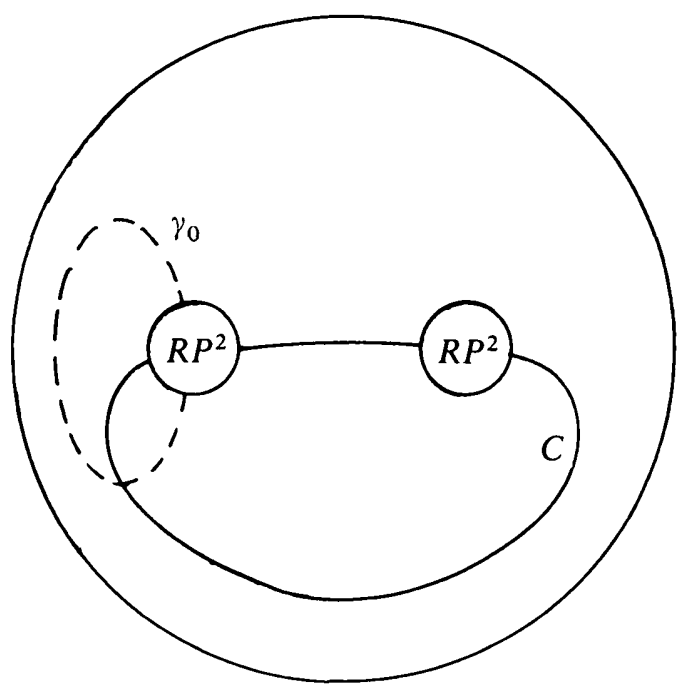

Figure 3. 


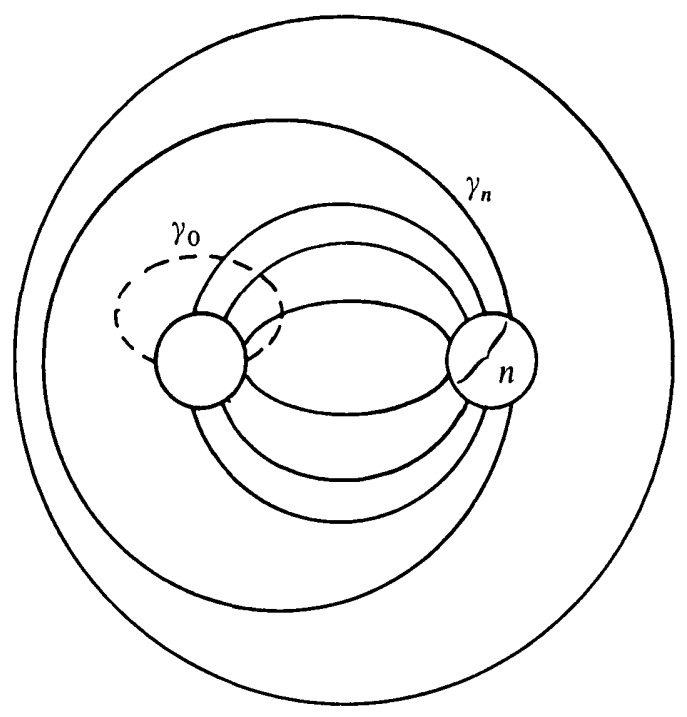

Figure 4.

Proof. Let $C$ be a simple closed 2-sided curve in the non-orientable surface $M$.

Case 1, when $C$ does not separate $M$. Let $N \subset M$ be the connected sum of a disk and two projective planes which contain $C$ and a curve $\gamma_{0}$ intersecting $C$ once (Figure 3). Let $\gamma_{n}$ be the curve obtained from $\gamma_{0}$ by twisting $n$ times along $C$ (Figure 4).

Evidently, no disk in $M-\left(\gamma_{n} \cup \gamma_{0}\right)$ has a boundary consisting of an interval in $\gamma_{n}$ and one in $\gamma_{0}$, and so the number of intersection points of $\gamma_{n}$ and $\gamma_{0}$ is minimized at $n-1$. It follows as in $[1 ; \S 3$.III] that any curve $\beta$ in $M$ can be isotoped to minimize simultaneously the number of intersection points with $\gamma_{0}$ and $\gamma_{n}$, so that $i\left(\beta, \gamma_{i}\right)=\#\left(\beta \cap \gamma_{i}\right), i=0, n$. Note that $\gamma_{0} \cup \gamma_{n}$ is the image of $n-1$ circles, of which $n-2$ are isotopic to $C$. Thus

$$
\sharp\left(\beta \cap\left(\gamma_{0} \cup \gamma_{n}\right)\right)=i\left(\beta, \gamma_{0}\right)+i\left(\beta, \gamma_{n}\right) \geqslant(n-2) i(\beta, C) .
$$

On the other hand, from the construction it follows that $i\left(\gamma_{n}, \beta\right)=\sharp\left(\gamma_{n} \cap \beta\right) \leqslant$ $n i(C, \beta)+i\left(\beta, \gamma_{0}\right)$. Thus

or

$$
n i(C, \beta)+i\left(\beta, \gamma_{0}\right) \geqslant i\left(\gamma_{n}, \beta\right) \geqslant(n-2) i(C, \beta)-i\left(\beta, \gamma_{0}\right)
$$

$$
\left|i\left(\gamma_{n}, \beta\right)-(n-1) i(C, \beta)\right| \leqslant i(C, \beta)+i\left(\beta, \gamma_{0}\right) .
$$

After projectivizing, $\lim _{n \rightarrow \infty} \gamma_{n}=C$ in $\mathscr{P}\left(R_{+}^{\mathscr{S}}-0\right)$.

Case 2, when $C$ separates $M$. The proof is analogous, with these alterations: $N$ is a 2-punctured projective plane and the curves $C, \gamma_{0}$ and $\gamma_{n}$ are as in Figure 5. The complex $\gamma_{0} \cup \gamma_{n}$ is the image of $2 n$ circles, $2 n-1$ of which are isotopic to $C$; the other is null-isotopic. 




Figure 5 .

\section{The elementary examples}

The examples covered in this section are

(1) the punctured Klein bottle $\left(\operatorname{dim}\left(\mathscr{P} \mathscr{F}_{0}\right)=1, \operatorname{dim}(\mathscr{P} \mathscr{F})=2\right)$,

(2) the 2-punctured projective plane $\left(\operatorname{dim}\left(\mathscr{P} \mathscr{F}_{0}\right)=0, \operatorname{dim}(\mathscr{P} \mathscr{F})=2\right)$,

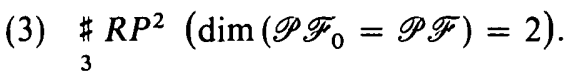

The only remaining non-orientable case in which $\operatorname{dim}(\mathscr{P} \mathscr{F}) \leqslant 2$ is the 3-punctured projective plane. It is substantially more complicated and is treated in the next section.

The punctured Klein bottle. On the (unpunctured) Klein bottle $K$ there are three isotopy classes of curves, $\bar{a}$ (two-sided) and $\bar{b}$ and $\bar{c}$ (one-sided), roughly corresponding to the generators $a, b$ and $a b=c$ of the fundamental group $\left\langle a, b: a b a b^{-1}=1\right\rangle$ (see Figure 6a). Equivalence classes of curves in the punctured Klein bottle (with puncture at a point $*$ ) then are represented to the images of $\bar{a}, \bar{b}$, and $\bar{c}$ under the isotopies of $K$ which carry $*$ back to itself, which in turn correspond to elements of $\pi_{1}(K)$. The curves in $K-*$ are thus the images of $\bar{a}, \bar{b}$ and $\bar{c}$ under the action of $\pi_{1}(K)$. (We might view $\pi_{1}(K)$ as the braid group of $K$ on one strand.) Since there is an isotopy from the identity $1_{K}: K \rightarrow K$ to itself which carries $*$ through the path $b^{2}$, this element of $\pi_{1}(K)$ acts trivially. Now the group $\pi_{1}(K) /\left\langle b^{2}\right\rangle$ is isomorphic to $\mathbb{Z}_{2} * \mathbb{Z}_{2}$ with generators $a b$ and $b$, and so any coset can be written as $a^{n}$ or $a^{n} b$, for $n$ an integer. Notice, finally, that the isotopy corresponding to $b$ has no effect on $\bar{b}$ or $\bar{a}$ (the latter merely reverses orientation) and carries $\bar{c}$ to $a^{-1}(\bar{c})$. Thus all simple closed curves can be obtained from $\bar{a}, \bar{b}$ and $\bar{c}$ by the action of $a$. Finally, note that a single Dehn twist $t$ about $\bar{a}$ has $t^{2}$ equivalent to $a$, and $t(\bar{b})=\bar{c}$. Thus in fact all curves can be obtained from $\bar{a}$ and $\bar{b}$ by the action of $t$. These are shown in Figure $6 \mathrm{~b}$. 
As $|n|$ becomes large, $t^{n}(b)$ approaches $\bar{a}$ in $\mathscr{P}\left(R_{+}^{\mathscr{S}}\right) . X_{0}$ can be viewed as lying on the circle $\mathscr{P} \mathscr{F}_{0}(M)$ as shown (Figure 7). The action of $b$ corresponds to reflection about the horizontal axis.

Note that each one-sided circle $t^{n}(\bar{b})$ is isolated, and the only 2-sided circle $\bar{a}$ is the limit of one-sided circles-a perfect illustration of Theorem 1.1 and Proposition 1.2. Also, $t^{n}(\bar{b})$ and $t^{n-1}(\bar{b})$ can be made disjoint on $K-*$, so they are joined by a 1 -simplex in $X_{0}$. Thus, in this case, $X_{0} \cong \mathscr{P} \mathscr{F}_{0}(M)$.

A similar analysis can be used on $X$. Any connected arc is the image of $a^{\prime}, b^{\prime}$ or $b^{\prime \prime}$ (Figure 8) under the action of $t$ (note that $b\left(b^{\prime \prime}\right)=t\left(b^{\prime \prime}\right)$ ).

The arcs in $K-*$ then are $\left\{a^{\prime}, t^{n}\left(b^{\prime}\right), t^{n}\left(b^{\prime \prime}\right) \mid n\right.$ an integer $\}$, and are shown in Figure 9. Note that each triple $\left\{\bar{b}, b^{\prime \prime}, t^{-1}(\bar{b})\right\}, \quad\left\{\bar{b}, b^{\prime}, b^{\prime \prime}\right\}, \quad\left\{b^{\prime}, b^{\prime \prime}, t^{-1}\left(b^{\prime}\right)\right\}$, $\left\{b^{\prime}, \bar{b}, t\left(b^{\prime \prime}\right)\right\}$ and $\left\{b^{\prime}, t^{-1}\left(b^{\prime}\right), a^{\prime}\right\}$ (and so all their translates by $t^{n}$ ) can be represented by disjoint arcs. An argument similar to that in Proposition 1.2 shows that $\lim t^{n}\left(b^{\prime}\right)=\bar{a}$. The resulting picture of $X \cong \mathscr{P} \mathscr{F}(M)$ is Figure 10 . $n \rightarrow \pm \infty$

The 2-punctured projective plane. The picture of $X$ is shown in Figure 11, together with illustrations of the circles and arcs representing each vertex. The leftand right-most vertices constitute $X_{0} \cong S^{0}$. (The small pictures are of $R P^{2}$ with 2-holes. The outer circle has antipodal points identified.)

Three projective planes. Another description is the connected sum of a torus and a projective plane. The projective plane contains a circle $C$ whose complement is orientable.
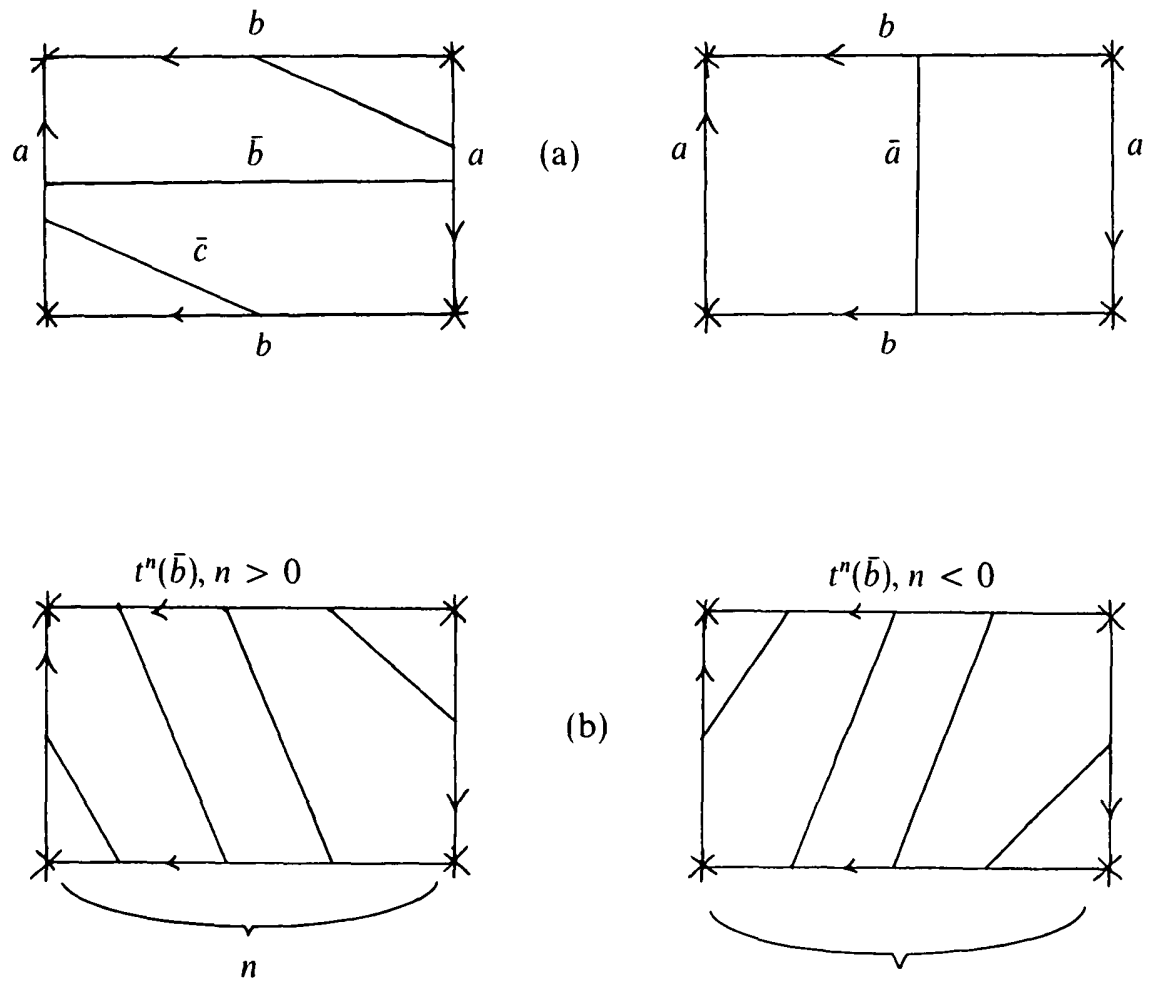

(a)

(b)

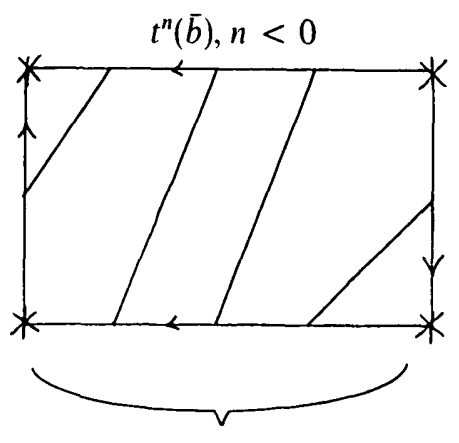

Figure 6.

$$
-n
$$




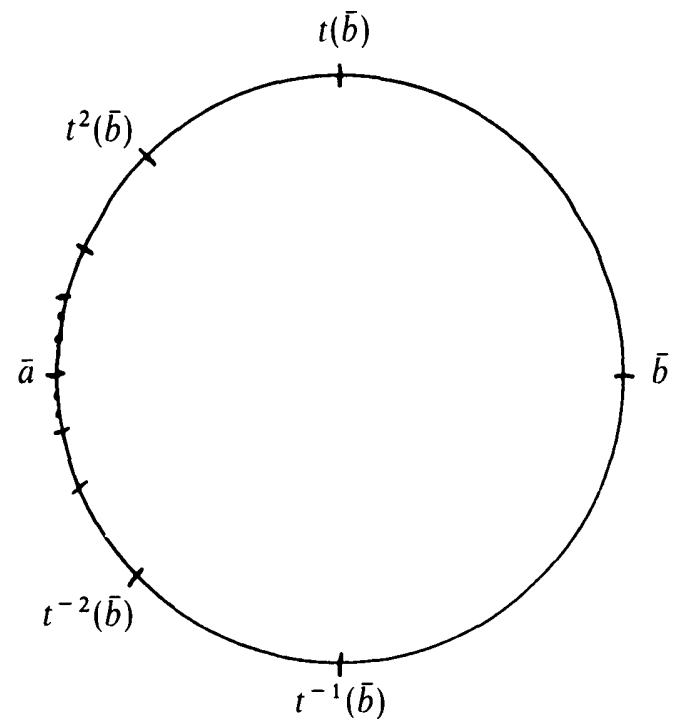

Figure 7.
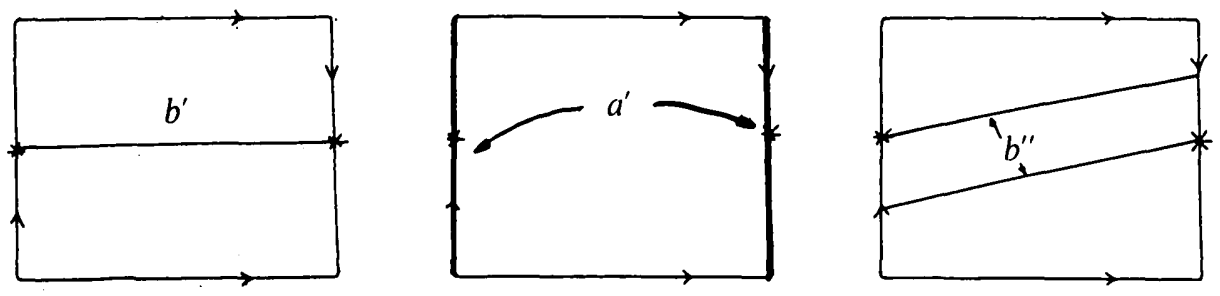

Figure 8.
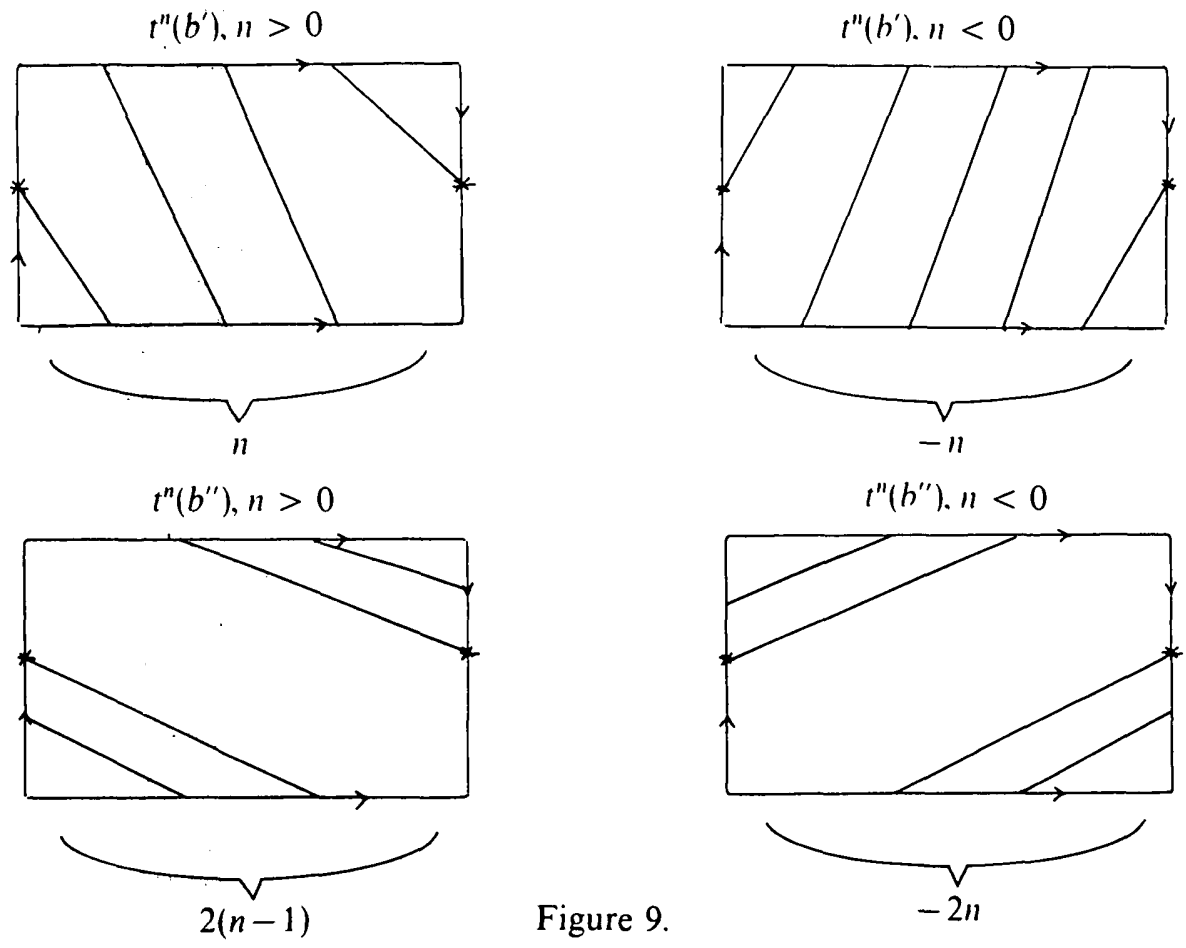

Figure 9.

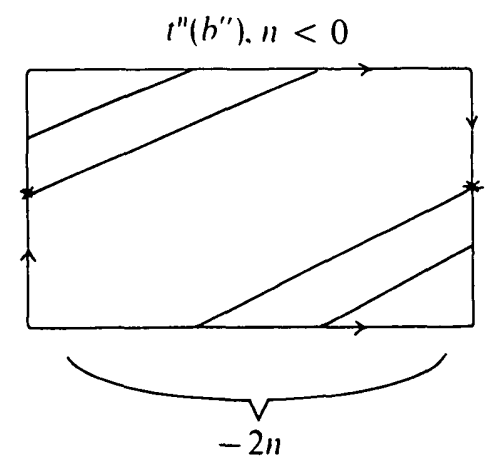




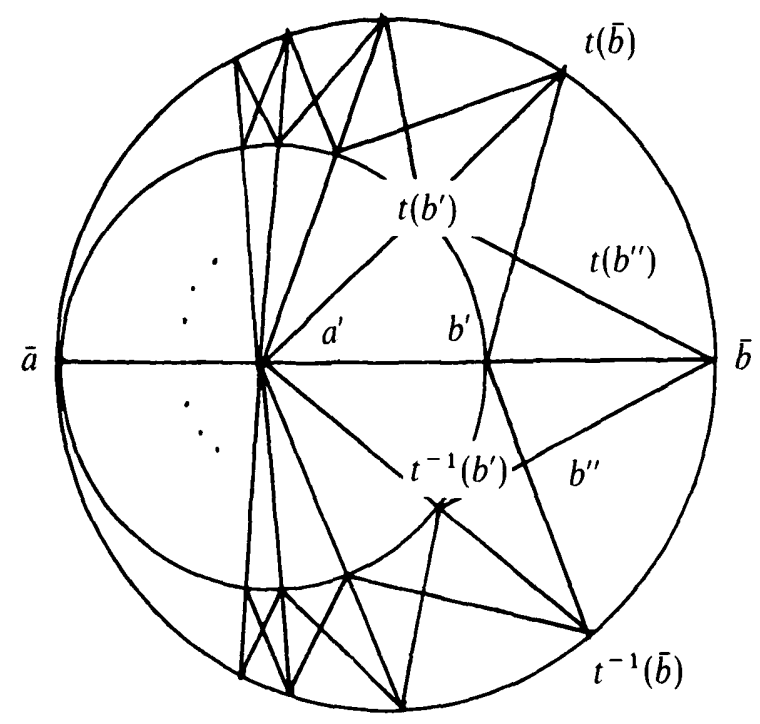

Figure 10.

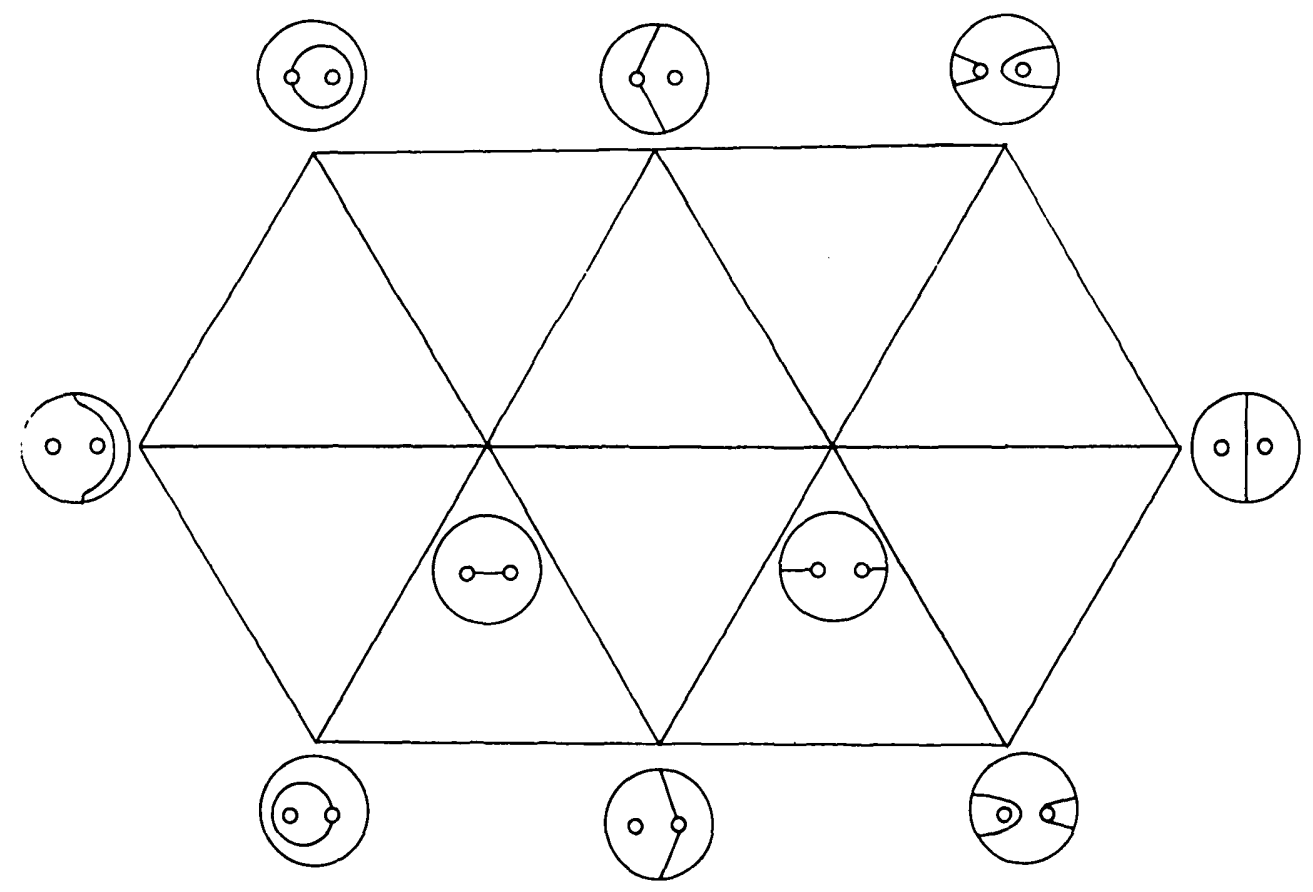

Figure 11. 


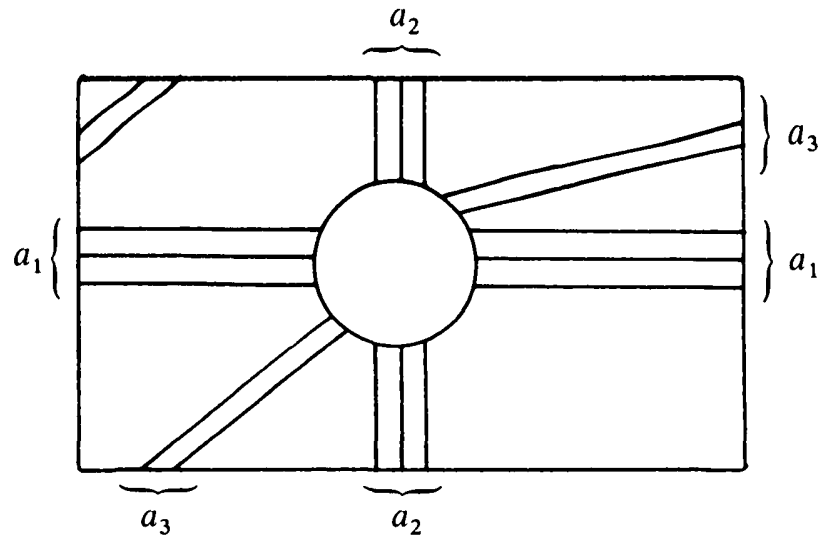

Figure 12.

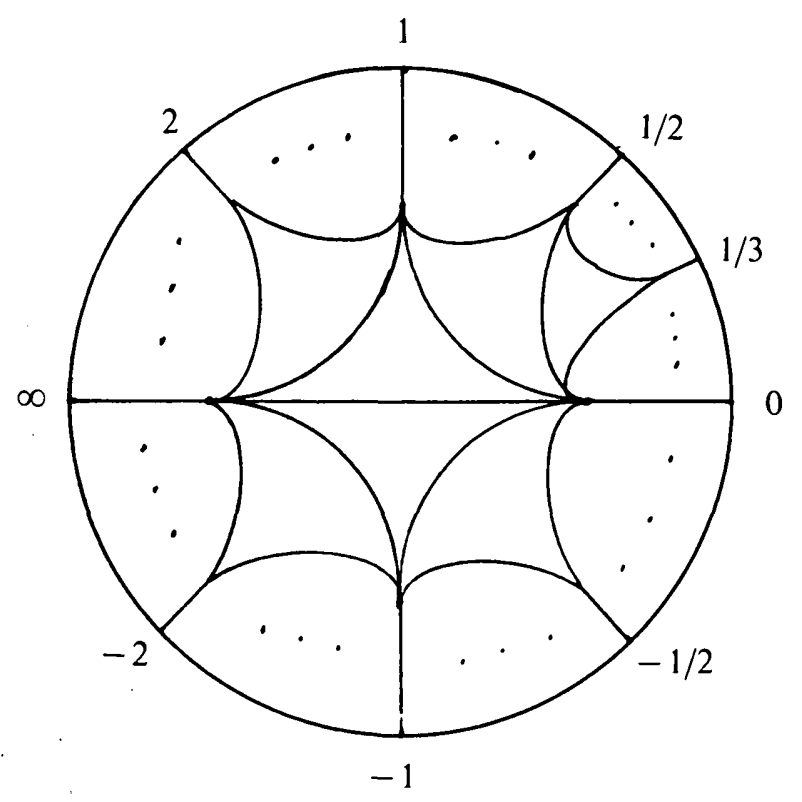

Figure 13. 
Lemma 2.1. Any simple closed curve in $M$ whose complement is orientable is isotopic to $C$.

Proof. Suppose that $s$ is a simple closed curve whose complement is orientable and which has been isotoped so that it intersects $C$ in a minimal number of points. Then in the punctured torus complement $N$ of $C, s$ is a non-trivial collection of arcs. After an automorphism of $N$, the intersection of $s$ with $N$ is as shown in Figure 12, with $a_{1} \geqslant a_{2} \geqslant a_{3}[2]$.

But since antipodal points of the boundary of $N$ are identified in $M$, and $s$ is connected, it follows that $a_{1} \leqslant 1, a_{2} \leqslant a_{3}=0$. When $a_{1}=1$, the complement of $s$ is non-orientable, so $a_{1}$ must also be trivial and so $s$ is equivalent to $C$.

Actually, the argument of Lemma 2.1 shows more. Except for the link of the vertex corresponding to $C$ in $X$, the complex $X$ is identical to the complex arising from the punctured torus. This has been studied by Floyd and Hatcher; it is the standard tiling of the disk by the action of $\operatorname{SL}(2, \mathbb{Z})$, except that the vertices are recessed away from the boundary of the disk, and whiskers run from the recessed vertices to the corresponding vertices on the boundary. An argument like that of Proposition 1.2 shows that the length of the whisker decreases with the area of the largest tile to which it belongs, as shown in Figure 13.

The (isolated) vertices in the interior of the disk correspond in $M$ to one-sided curves intersecting $C$, those in the boundary to two-sided curves disjoint from $C$. The complete picture of $X$ is then that of Figure 14, together with the cone (to the vertex corresponding to $C$ ) of all the vertices on the boundary of the disk. This is the first example in which $X \subsetneq \mathscr{P} \mathscr{F}(M)$, for in the hemisphere in which $X$ consists of the cone on the rationals, there are no two-simplices in $X$.

\section{The 3-punctured projective plane}

This is the final and most complicated example. It is simplified by observing that in the complement of any two-sided (non-boundary parallel) simple closed curve there are exactly two non-isotopic one-sided curves, which intersect in a single point. Conversely, any two non-isotopic one-sided curves which intersect once have exactly one non-boundary parallel two-sided curve in their complement. In $X$, this means that vertices representing two-sided curves appear precisely as interior points of edges connecting those vertices in $X$ representing one-sided curves which intersect once in $M$. Thus to find $X$ it suffices to find $Y$, the 1 -dimensional subcomplex of $\mathscr{P P} \mathscr{F}(M)$ whose vertices represent one-sided curves and whose edges connect those vertices whose curves intersect precisely once.

THEOREM 3.1. $\quad Y$ is the 1-skeleton of the complex obtained from a tetrahedron by repeated stellar subdivision of the faces, but not the edges.

Proof. The easy part is to see the original tetrahedron; Figure 14 shows four distinct one-sided curves $s_{1}, s_{2}, s_{3}, s_{4}$ in $M$, each pair of which intersects in precisely one point. (Once again, $R P^{2}$ is represented as a disk with antipodal boundary points identified.)

In order to locate the vertex corresponding to a given one-sided curve in the barycentric subdivision of this tetrahedron, associate to a vertex $x$ a "length" as 


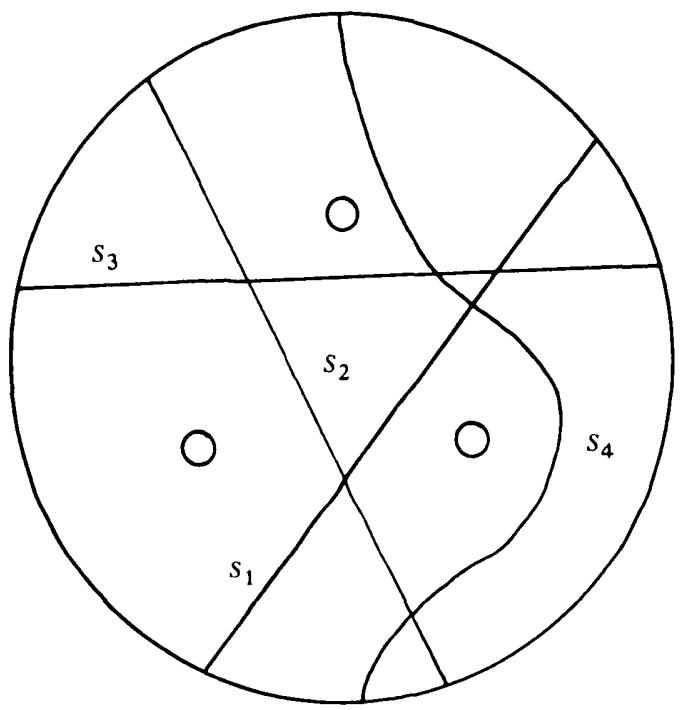

Figure 14.

follows (the length will roughly correspond to the number of subdivisions necessary before reaching the vertex). Choose an automorphism $f: M \rightarrow M$ which carries $x$ to one of the $s_{i}, 1 \leqslant i \leqslant 4$. Then $f$ extends to an automorphism $\bar{f}: R P^{2} \rightarrow R P^{2}$ which must be isotopic to the identity. The image of the three punctures under such an isotopy is an element $w$ of the braid group $B_{3}\left(R P^{2}\right)$. The group $B_{3}(R P)^{2}$ has a presentation given by the generators $\sigma_{1}, \sigma_{2}, \sigma_{3}, r_{1}, r_{2}, r_{3}$ as shown (Figure 15) and the relations $(j \equiv i+1, k \equiv j+1 \bmod 3)$

(i) $\sigma_{j} \sigma_{i}=\sigma_{k} \sigma_{j}$,

(ii) $r_{k} \sigma_{i}=\sigma_{i} r_{k}$,

(iii) $r_{j}=\sigma_{i}^{-1} r_{i} \sigma_{i}$,

(iv) $r_{i} r_{j}^{-1} r_{i}^{-1} r_{j}=\sigma_{i}^{2}$,

(v) $r_{j}^{2}=\sigma_{j}^{2} \sigma_{i}^{2}$

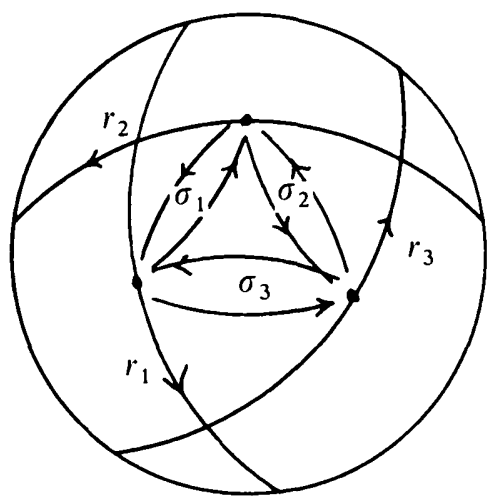

Figure 15. 
(see [4] and make the substitutions $\sigma_{3}=\sigma_{1} \sigma_{2} \sigma_{1}^{-1}, r_{1}=\rho_{1}, r_{3}=\rho_{3}^{-1}$, $r_{2}=\sigma_{1} r_{1} \sigma_{1}^{-1}$ so that the generators have a 3-fold symmetry corresponding to adding $1(\bmod 3)$ to all subscripts; then compose from right to left, as functions are composed).

Denote the minimal length of $w$ written in the generators $\sigma_{i}, r_{i}$ by $l(f)$. Finally, the length $l(x)=\min _{f \mid S(x) \in\left\{s_{i}\right\}} l(f)$. In particular $l(x)=0$ if and only if $x \in\left\{s_{i}\right\}$.

Definition. $Y_{n}$ is the 1-dimensional subcomplex of $Y$ consisting of vertices of length $\leqslant n$ and the edges joining them. The closure of a component of $\mathscr{P} \mathscr{F}-Y_{n}$ will be called a face of $Y_{n}$ (though it does not belong to $Y_{n}$ ).

It would be pleasant to prove that $Y_{n}$ is obtained from $Y_{n-1}$ by stellar subdivision of each face; the proof of Theorem 3.1 would then be immediate. Unfortunately, this fails (for example $Y_{1}$ contains only three of the four centres of the tetrahedron's faces (see below)) but, exploiting the topology of $\mathscr{P} \mathscr{F} \cong S^{2}$ in which $Y_{n}$ lies, there is the following weaker result.

Proposition 3.2. (1) $Y_{n+1}$ is obtained from $Y_{n}$ by stellar subdivision of some faces.

(2) For any face in $Y_{n}$ there is an $m>n$ such that the intersection of $Y_{m}$ with that face is its stellar subdivision.

Theorem 3.1 then follows immediately.

Lemma 3.3. Any vertex $x$ of $Y$ with $l(x)>0$ lies in a tetrahedral 1-complex whose other three vertices have shorter length.

Proof. Throughout the proof $1 \leqslant i, j, k \leqslant 3$ and $k \equiv j+1 \equiv i+2(\bmod 3)$. From Figure 15 these follow:
(a) $\sigma_{i}\left(s_{i}\right)=s_{i}$
(b) $\sigma_{i}\left(s_{k}\right)=s_{j}$;
(c) $\sigma_{i}\left(s_{4}\right)=s_{4}$;

(d) $r_{i}\left(s_{j}\right)=s_{4}$;

(e) $r_{i}\left(s_{k}\right)=s_{i}$;

(f) $r_{i}\left(s_{4}\right)=s_{j}$;

(g) $\sigma_{k}^{-1}\left(s_{j}\right)=r_{i}^{-1}\left(s_{k}\right)$

In particular, the only three vertices in $Y_{1}$ not in $Y_{0}$ are the $\sigma_{i}^{\prime}\left(s_{j}\right)=\sigma_{j}^{-1}\left(s_{i}^{\prime}\right)$.

Suppose now that $w$ is the word of minimal length such that $w\left(s_{l}\right)=x$, for some $1 \leqslant l \leqslant 4$, and $g$ the last symbol in $w$, so that $w=\bar{w} g$ for some $\bar{w}$. By (c), (d) and (f), $w$ can only be shortest if $l \neq 4$, and so we may write $s_{l}$ as $s_{i}$. Then, by (a) and (b), $g \neq \sigma_{i}, \sigma_{j}, \sigma_{i}^{-1}, \sigma_{k}^{-1}$. By (d), (e), and (f), $g \neq r_{j}, r_{k}, r_{i}^{-1}, r_{k}^{-1}$. We may further assume that $g \neq r_{j}^{-1}$ or $r_{i}$, for $r_{i}\left(s_{i}\right)=r_{i}^{2}\left(s_{k}\right)=\sigma_{i}^{2} \sigma_{k}^{2}\left(s_{k}\right)=\sigma_{i}^{2}\left(s_{k}\right)=\sigma_{i}\left(s_{j}\right)$ and $r_{j}^{-1}\left(s_{i}\right)=r_{j}^{-1} \sigma_{k}\left(s_{j}\right)=\sigma_{k} r_{j}^{-1}\left(s_{j}\right)=\sigma_{k}\left(s_{i}\right)$. If $g=\sigma_{k}$ then $\bar{w} g\left(s_{j}\right)=\bar{w}\left(s_{i}\right)$, $\bar{w} g\left(s_{k}\right)=\bar{w}\left(s_{k}\right)$ and $\bar{w} g\left(s_{4}\right)=\bar{w}\left(s_{4}\right)$ are three "shorter" vertices of $\cdot$ a tetrahedral 1-complex containing $\bar{w} g\left(s_{i}\right)=x$. If $g=\sigma_{j}^{-1}$, then $\bar{w} g\left(s_{j}\right)=\bar{w}\left(s_{j}\right), \bar{w} g\left(s_{k}\right)=\bar{w}\left(s_{i}\right)$, and $\bar{w} g\left(s_{4}\right)=\bar{w}\left(s_{4}\right)$ are the required vertices.

Corollary 3.4. $Y_{n}$ is connected.

Proof. Use induction on $n$.

COROLLARY 3.5. Any triangle in $Y_{n}$ containing a vertex of length $n$ bounds a single face of $Y_{n}$. 


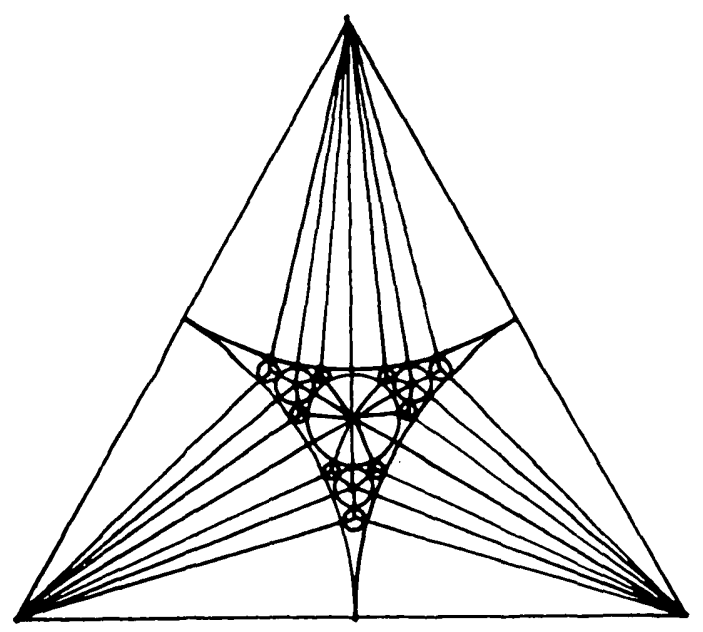

Figure 16.

Proof. Let $\triangle$ be a triangle in $Y_{n}$ and $x$ a vertex in $\Delta$ of length $n$. The triangle $\Delta$ divides $\mathscr{P} \mathscr{F} \cong S^{2}$ into two components. If a component contains a vertex of $Y_{n}$ then, by Lemma 3.3, it contains a vertex of length zero, that is, an $s_{i}$. But since all the $s_{i}$ are connected in $Y_{0}$, they must all be in the same component. Thus one component contains no vertex of $Y_{n}$ and so is a face. The other component contains an $s_{i}$ and so is not a face.

Proof of Proposition 3.2. To prove (1) note that any vertex $v$ of length $n+1$ is connected, by Lemma 3.3, to three vertices of a triangle $x_{1}, x_{2}, x_{3}$ in $Y_{n}$. By Corollary 3.5 each triangle with vertices $\left\{v, x_{i}, x_{j}\right\}$ bounds a face of $Y_{n+1}$. Thus the original triangle $\left\{x_{1}, x_{2}, x_{3}\right\}$ bounds a face of $Y_{n}$ (the union of these faces of $Y_{n+1}$ ) in which $v$ lies, and $Y_{n+1}$ stellar subdivides this face.

To prove (2), suppose that $\left\{x_{1}, x_{2}, x_{3}\right\}$ are vertices of a triangle bounding a face $F$ of $Y_{n}$. Then there is a word $w$ in $B_{3}\left(R P^{2}\right)$ such that $w\left(s_{i}\right)=x_{i}, i=1,2,3$. Both the vertices $w\left(s_{4}\right)$ and $w \sigma_{3} r_{1}^{-1} \sigma_{3}\left(s_{1}\right)$ are connected to each $w\left(s_{i}\right)$, for $w\left(s_{1}\right)=w \sigma_{3} r_{1}^{-1} \sigma_{3}\left(s_{4}\right), w\left(s_{2}\right)=w \sigma_{3} r_{1}^{-1} \sigma_{3}\left(s_{3}\right)$ and $w\left(s_{3}\right)=w \sigma_{3} r_{1}^{-1} \sigma_{3}\left(s_{2}\right)$, so that either $w\left(s_{4}\right)$ or $w \sigma_{3} r_{1}^{-1} \sigma_{3}\left(s_{1}\right)$ lies in $F$ and, by definition of face, has length $m>n$. The argument of part (1) shows that no other vertex of $Y_{m}$ lies in $F$, and so $Y_{m}$ intersects $F$ in its stellar subdivision.

Remark 3.6. Figure 16 (a picture of the Apollonian packing, suggested by Allen Hatcher) shows a subdivision as in Theorem 3.1 which does satisfy Theorem 1.1, based on an infinity of nested circles. Vertices on circles represent 2 -sided curves. Centres of circles represent 1 -sided curves. Only one face of the tetrahedron is shown.

\section{References}

1. A. Fathi, F. Laudenbach and V. Poenaru, "Travaux de Thurston sur les surfaces", Asterisque, 66-67 (1979), 1-284.

2. W. Floyd and A. Hatcher, "Curves on the 4-punctured sphere; 31 charts", preprint, University of California at Los Angeles, 1980. 
3. W. Thurston, "On the geometry and dynamics of diffeomorphisms of surfaces", preprint, Princeton University, 1976.

4. J. Van Buskirk, "Braid groups of compact 2-manifolds with elements of finite order"; Trans. Amer. Math. Soc., 122 (1966), 81-97.

Department of Mathematics, University of California, Santa Barbara,

California 93106, U.S.A. 